\title{
An Interesting Property of a Class of Circulant Graphs
}

\author{
Seyed Morteza Mirafzal and Ali Zafari \\ Department of Mathematics, Lorestan University, Khoramabad, Iran \\ Correspondence should be addressed to Ali Zafari; zafari.ali@fs.lu.ac.ir
}

Received 22 July 2016; Accepted 22 January 2017; Published 27 February 2017

Academic Editor: Michel Bauer

Copyright (C) 2017 Seyed Morteza Mirafzal and Ali Zafari. This is an open access article distributed under the Creative Commons Attribution License, which permits unrestricted use, distribution, and reproduction in any medium, provided the original work is properly cited.

\begin{abstract}
Suppose that $\Pi=\operatorname{Cay}\left(\mathbb{Z}_{n}, \Omega\right)$ and $\Lambda=\operatorname{Cay}\left(\mathbb{Z}_{n}, \Psi_{m}\right)$ are two Cayley graphs on the cyclic additive group $\mathbb{Z}_{n}$, where $n$ is an even integer, $m=n / 2+1, \Omega=\left\{t \in \mathbb{Z}_{n} \mid t\right.$ is odd $\}$, and $\Psi_{m}=\Omega \cup\{n / 2\}$ are the inverse-closed subsets of $\mathbb{Z}_{n}-\{0\}$. In this paper, it is shown that $\Pi$ is a distance-transitive graph, and, by this fact, we determine the adjacency matrix spectrum of $\Pi$. Finally, we show that if $n \geq 8$ and $n / 2$ is an even integer, then the adjacency matrix spectrum of $\Lambda$ is $(n / 2+1)^{1},(1-n / 2)^{1},(1)^{(n-4) / 2},(-1)^{n / 2}$ (we write multiplicities as exponents).
\end{abstract}

\section{Introduction}

In this paper, graph $\Gamma=(V, E)$ always means a simple connected graph with $n$ vertices (without loops, multiple edges, and isolated vertices), where $V=V(\Gamma)$ is the vertex set and $E=E(\Gamma)$ is the edge set. Graph $\Gamma$ is called a vertextransitive graph, if, for any $x, y \in V$, there is some $\pi$ in $\operatorname{Aut}(\Gamma)$, the automorphism group of $\Gamma$, such that $\pi(x)=y$. Let $\Gamma$ be a graph, the complement $\bar{\Gamma}$ of $\Gamma$ is the graph whose vertex set is $V(\Gamma)$ and whose edges are the pairs of nonadjacent vertices of $\Gamma$. It is well known that, for any graph $\Gamma, \operatorname{Aut}(\Gamma)=\operatorname{Aut}(\bar{\Gamma})[1]$. If $\Gamma$ is a connected graph and $\partial(u, v)$ denotes the distance in $\Gamma$ between the vertices $u$ and $v$, then, for any automorphism $\pi$ in $\operatorname{Aut}(\Gamma)$, we have $\partial(u, v)=\partial(\pi(u), \pi(v))$.

Let $\Upsilon=\left\{\gamma_{1}, \ldots, \gamma_{k+1}\right\}$ be a set and $K$ be a group; then, writing Fun $(Y, K)$ to denote the set of all functions from $Y$ into $K$, we can turn $\operatorname{Fun}(\Upsilon, K)$ into a group by defining a product:

$$
\begin{aligned}
&(f g)(\gamma)=f(\gamma) g(\gamma) \\
& \forall f, g \in \operatorname{Fun}(\Upsilon, K) \text { and } \gamma \in \Upsilon,
\end{aligned}
$$

where the product on the right is in $K$. Since $\Upsilon$ is finite then the group $\operatorname{Fun}(\Upsilon, K)$ is isomorphic to $K^{k+1}$ (a direct product of $k+1$ copies of $K$ ) via the isomorphism $f \rightarrow$ $\left(f\left(\gamma_{1}\right), \ldots, f\left(\gamma_{k+1}\right)\right)$. Let $H$ and $K$ be groups and suppose that $H$ acts on the nonempty set $\Upsilon$. Then, the wreath product of $K$ by $H$ with respect to this action is defined to be the semidirect product $\operatorname{Fun}(\Upsilon, K) \rtimes H$ where $H$ acts on the group $\operatorname{Fun}(\Upsilon, K)$ via

$$
\begin{aligned}
& f^{x}(\gamma)=f\left(\gamma^{x^{-1}}\right) \\
& \quad \forall f \in \operatorname{Fun}(\Upsilon, K), \gamma \in \Upsilon \text { and } x \in H .
\end{aligned}
$$

We denote this group by $\mathrm{Kwr}_{\Upsilon} H$. Consider the wreath product $G=K w_{\Upsilon} H$. If $K$ acts on a set $\Delta$, then we can define an action of $G$ on $\Delta \times \Upsilon$ by

$$
(\delta, \gamma)^{(f, h)}=\left(\delta^{f(\gamma)}, \gamma^{h}\right) \quad \forall(\delta, \gamma) \in \Delta \times \Upsilon,
$$

where $(f, h) \in \operatorname{Fun}(Y, K) \rtimes H=K \mathrm{wr}_{\Upsilon} H[2]$.

Let $G$ be a group and $H \leq G$ a subgroup of $G$ and $S \subseteq G$. The Schreier coset graph on $G / H$ generated by $S$ is the graph $\Gamma=\Gamma(G, H, S)$ with $V(\Gamma)=G / H=\{g H \mid g \in G\}$ the set of left cosets of $H$, and there is an edge $(g H, s g H)$ for each coset $g H$ and each $s \in S$. If $S$ is inverse-closed, then $\Gamma$ is an undirected multigraph (possibly with loops). Note that if $1_{G}$ is the identity element of $G$, then $\Gamma\left(G, 1_{G}, S\right)=\Gamma(G, S)$ is the Cayley graph on $G$ generated by $S$. It is well known that every Cayley graph is vertex-transitive [3].

Let $\Gamma$ be a graph with automorphism group $\operatorname{Aut}(\Gamma)$. Say that $\Gamma$ is symmetric graph if, for all vertices $u, v, x, y$ of $\Gamma$ such that $u$ and $v$ are adjacent, also, $x$ and $y$ are adjacent, and there is an automorphism $\pi$ in $\operatorname{Aut}(\Gamma)$ such that $\pi(u)=x$ 
and $\pi(v)=y$. We say that $\Gamma$ is distance-transitive if, for all vertices $u, v, x, y$ of $\Gamma$ such that $\partial(u, v)=\partial(x, y)$, there is an automorphism $\pi$ in $\operatorname{Aut}(\Gamma)$ satisfying $\pi(u)=x$ and $\pi(v)=y$ [3]. It is clear that hierarchy of the conditions is

$$
\begin{aligned}
\text { distance-transitive } & \Longrightarrow \text { symmetric } \\
& \Longrightarrow \text { vertex-transitive. }
\end{aligned}
$$

Eigenvalues of an undirected graph $\Gamma$ are the eigenvalues of an arbitrary adjacency matrix of $\Gamma$. Harary and Schwenk [4] defined $\Gamma$ to be integral, if all of its eigenvalues are integers. For a survey of integral graphs, see [5]. In [6], the number of integral graphs on $n$ vertices is estimated. Known characterizations of integral graphs are restricted to certain graph classes; see [7].

In this paper, suppose $\Pi=\operatorname{Cay}\left(\mathbb{Z}_{n}, \Omega\right)$ and $\Lambda=$ $\operatorname{Cay}\left(\mathbb{Z}_{n}, \Psi_{m}\right)$ are two Cayley graphs on the cyclic additive group $\mathbb{Z}_{n}$, where $n$ is an even integer, $m=n / 2+1, \Omega=$ $\left\{t \in \mathbb{Z}_{n} \mid t\right.$ is odd $\}$, and $\Psi_{m}=\Omega \cup\{n / 2\}$ are the inverseclosed subsets of $\mathbb{Z}_{n}-\{0\}$. One of our goals in this paper is to obtain all eigenvalues of the Cayley graph $\Lambda=\operatorname{Cay}\left(\mathbb{Z}_{n}, \Psi_{m}\right)$. First, we determine the group automorphism of $\Pi$ and we show that $\Pi$ is a distance transitive graph; also, by this fact, we determine the adjacency matrix spectrum of $\Pi$. Finally, according to these facts, we show that if $n \geq 8$ and $n / 2$ is an even integer, then the adjacency matrix spectrum of $\Lambda$ is $(n / 2+1)^{1},(1-n / 2)^{1},(1)^{(n-4) / 2},(-1)^{n / 2}$ (we write multiplicities as exponents).

\section{Definitions and Preliminaries}

Definition 1 (see $[3,8])$. For any vertex $v$ of a connected graph $\Gamma$, one defines

$$
\Gamma_{r}(v)=\{u \in V(\Gamma) \mid \partial(u, v)=r\},
$$

where $r$ is a nonnegative integer not exceeding $d$, the diameter of $\Gamma$. It is clear that $\Gamma_{0}(v)=\{v\}$, and $V(\Gamma)$ is partitioned into the disjoint subsets $\Gamma_{0}(v), \ldots, \Gamma_{d}(v)$, for each $v$ in $V(\Gamma)$. The graph $\Gamma$ is called distance-regular with diameter $d$ and intersection array $\left\{b_{0}, \ldots, b_{d-1} ; c_{1}, \ldots, c_{d}\right\}$, if it is regular of valency $k$ and, for any two vertices $u$ and $v$ in $\Gamma$ at distance $r$, one has $\mid \Gamma_{r+1}(v) \cap$ $\Gamma_{1}(u) \mid=b_{r}$, and $\left|\Gamma_{r-1}(v) \cap \Gamma_{1}(u)\right|=c_{r},(0 \leq r \leq d)$. The numbers $c_{r}, b_{r}$, and $a_{r}$, where

$$
a_{r}=k-b_{r}-c_{r} \quad(0 \leq r \leq d),
$$

is the number of neighbours of $u$ in $\Gamma_{r}(v)$ for $\partial(u, v)=r$, are called the intersection numbers of $\Gamma$. Clearly $b_{0}=k, b_{d}=c_{0}=$ 0 , and $c_{1}=1$.

Remark 2 (see [3]). It is clear that if $\Gamma$ is distance-transitive graph, then $\Gamma$ is distance-regular.

Lemma 3 (see [3]). A connected graph $\Gamma$ with diameter $d$ and automorphism group $G=\operatorname{Aut}(\Gamma)$ is distance-transitive if and only if it is vertex-transitive and the vertex-stabilizer $G_{v}$ is transitive on the set $\Gamma_{r}(v)$, for each $r \in\{0,1, \ldots, d\}$, and $v \in V(\Gamma)$.
Theorem 4 (see [8]). Let $\Gamma$ be a distance-regular graph which the valency of each vertex as $k$, with diameter $d$, adjacency matrix $A$, and intersection array, is

$$
\left\{b_{0}, b_{1}, \ldots, b_{d-1} ; c_{1}, c_{2}, \ldots, c_{d}\right\} .
$$

Then, the tridiagonal $(d+1) \times(d+1)$ matrix

$$
j(\Gamma)=\left[\begin{array}{cccccccc}
a_{0} & b_{0} & 0 & 0 & \cdots & & & \\
c_{1} & a_{1} & b_{1} & 0 & \ldots & & & \\
0 & c_{2} & a_{2} & b_{2} & & & & \\
& & & \cdots & & & & \\
& & & & c_{d-2} & a_{d-2} & b_{d-2} & 0 \\
& & & \ldots & 0 & c_{d-1} & a_{d-1} & b_{d-1} \\
& & & \ldots & 0 & 0 & c_{d} & a_{d}
\end{array}\right]
$$

determines all the eigenvalues of $\Gamma$.

Theorem 5 (see [9]). Let $F$ be a field and let $R$ be $a$ commutative subring of $F^{n \times n}$, the set of all $n \times n$ matrices over F. Let $M \in R^{m \times m}$, then $\operatorname{det}_{F}(M)=\operatorname{det}_{F}\left(\operatorname{det}_{R}(M)\right)$.

Theorem 6 (see [10]). Let $\Gamma$ be a graph such that contains $k+1$ components $\Gamma_{1}, \ldots, \Gamma_{k+1}$. If, for any $i \in I=\{1, \ldots, k+1\}, \Gamma_{i} \cong$ $\Gamma_{1}$, then $\operatorname{Aut}(\Gamma) \cong \operatorname{Aut}\left(\Gamma_{1}\right) w r_{I} \operatorname{Sym}(k+1)$.

\subsection{Main Results}

Proposition 7. Let $\Pi=\operatorname{Cay}\left(\mathbb{Z}_{n}, \Omega\right)$ be the Cayley graph on the cyclic group $\mathbb{Z}_{n}(n \geq 4)$, where $\Omega=\left\{t \in \mathbb{Z}_{n} \mid\right.$ $t$ is odd $\}$ is the inverse-closed subset of $\mathbb{Z}_{n}-\{0\}$. Then $\operatorname{Aut}(\Pi) \cong$ $\operatorname{Sym}(n / 2) w r_{I} \operatorname{Sym}(2)$, where $I=\{1,2\}$.

Proof. Let $V(\Pi)=\{1, \ldots, n\}$ be the vertex set of $\Pi$. By assumption, the size of the every independent set of vertices in $\Pi$ is $n / 2$, because $\Pi$ is a vertex-transitive graph and the size of every clique in graph $\Pi$ is 2 . Therefore, for any $x \in V(\Pi)$, there is exactly $n / 2, y \in V(\Pi)$ such that $x^{-1} y \notin \Omega$. Hence, if $x^{-1} y \notin \Omega$, then two vertices $x$ and $y$ are adjacent in the complement $\bar{\Pi}$ of $\Pi$, so $\bar{\Pi}$ contains 2 components $\Pi_{1}, \Pi_{2}$ such that $\Pi_{1} \cong \Pi_{2} \cong K_{n / 2}$, where $K_{n / 2}$ is the complete graph of $n / 2$ vertices. Therefore, $\bar{\Pi} \cong 2 K_{n / 2}$. Hence, by Theorem 6 , $\operatorname{Aut}(\bar{\Pi}) \cong \operatorname{Aut}\left(K_{n / 2}\right) \mathrm{wr}_{I} \operatorname{Sym}(2)=\operatorname{Sym}(n / 2) \mathrm{wr}_{I} \operatorname{Sym}(2)$.

Proposition 8. Let $\Pi=\operatorname{Cay}\left(\mathbb{Z}_{n}, \Omega\right)$ be the Cayley graph on the cyclic group $\mathbb{Z}_{n}(n \geq 4)$, where $n$ is an even integer and $\Omega=\left\{t \in \mathbb{Z}_{n} \mid t\right.$ is odd $\}$ is the inverse-closed subset of $\mathbb{Z}_{n}-\{0\}$; then $\Pi$ is a distance-transitive graph.

Proof. Suppose that $u, v, x, y$ are vertices of $\Pi$ such that $\partial(u, v)=\partial(x, y)=r$, where $r$ is a nonnegative integer not exceeding $d$, the diameter of $\Pi$. So $\partial(u, v)=\partial(x, y)=1$ or 2 , since $d=2$.

(a) If $\partial(u, v)=\partial(x, y)=2$, then $u^{-1} v \notin \Omega$ and $x^{-1} y \notin \Omega$. Therefore, two vertices $u$ and $v$ are adjacent in the complement $\bar{\Pi}$ of $\Pi$, also two vertices $x$ and $y$ are adjacent in the complement $\bar{\Pi}$ of $\Pi$. So $\bar{\Pi}$ contains 2 components $\Pi_{1}, \Pi_{2}$ 
such that $\Pi_{1} \cong \Pi_{2} \cong K_{n / 2}$. Therefore $\bar{\Pi} \cong 2 K_{n / 2}$; hence we may assume $\pi=(u x)(v y) \in \operatorname{Aut}(\bar{\Pi})=\operatorname{Aut}(\Pi)$, so $\pi(u)=x$ and $\pi(v)=y$.

(b) If $\partial(u, v)=\partial(x, y)=1$, then, by Lemma 3, it is sufficient to show that vertex-stabilizer $G_{v}$ is transitive on set $\Pi_{r}(v)$ for every $r \in\{0,1,2\}$ and every $v \in V(\Pi)$, because $\Pi$ is a vertex-transitive graph. In this case, let $V(\Pi)=\{1,2, \ldots, n\}$ be the vertex set of $\Pi$ and $G=\operatorname{Aut}(\Pi)$. Consider the vertex $v=1$ in $V(\Pi)$, then $\Pi_{0}(v)=\{1\}, \Pi_{1}(v)=\left\{t \in \mathbb{Z}_{n} \mid t\right.$ is even $\}$, and $\Pi_{2}(v)=\left\{1 \neq t \in \mathbb{Z}_{n} \mid t\right.$ is odd $\}$. Let $H$ be the group that is generated by all elements of sets $\Pi_{1}(v)$ and $\Pi_{2}(v)$, say $H=\langle(2,4, \ldots, n),(3,5, \ldots, n-1)\rangle$. It is clear that $H$ is a subgroup of $\operatorname{Aut}(\Pi)$, so the group $H$ is a subgroup of $G_{v}$ such that transitive on the set $\Pi_{r}(v)$ for each $r \in\{0,1,2\}$. Note that if $1 \neq v \in V(\Pi)$, then, we can show that vertex-stabilizer $G_{v}$ is transitive on the set $\Pi_{r}(v)$ for each $r \in\{0,1,2\}$, because $\Pi$ is a vertex-transitive graph.

Proposition 9. Let $\Pi=\operatorname{Cay}\left(\mathbb{Z}_{n}, \Omega\right)$ be the Cayley graph on the cyclic group $\mathbb{Z}_{n}(n \geq 4)$, where $n$ is an even integer and $\Omega=\left\{t \in \mathbb{Z}_{n} \mid t\right.$ is odd $\}$ is the inverse-closed subset of $\mathbb{Z}_{n}-\{0\}$; then $\Pi$ is an integral graph.

Proof. By Remark 2, it is clear that $\Pi$ is distance-regular, because $\Pi$ is a distance-transitive graph. Let $V(\Pi)=$ $\{1,2, \ldots, n\}$ be the vertex set of $\Pi$. Consider the vertex $v=1$ in $V(\Pi)$; then $\Pi_{0}(v)=\{1\}, \Pi_{1}(v)=\left\{t \in \mathbb{Z}_{n} \mid t\right.$ is even $\}$, and $\Pi_{2}(v)=\left\{1 \neq t \in \mathbb{Z}_{n} \mid t\right.$ is odd $\}$. Let be $u$ in $V(\Pi)$ such that $\partial(u, v)=0$; then $u=v=1$ and $\left|\Pi_{1}(v) \cap \Pi_{1}(u)\right|=n / 2$; hence $b_{0}=n / 2$ and, by Definition $1, a_{0}=n / 2-b_{0}=0$. Also, if $u$ in $V(\Pi)$ and $\partial(u, v)=1$, then two vertices $u, v$ are adjacent in $\Pi$, so $\left|\Pi_{0}(v) \cap \Pi_{1}(u)\right|=1$ and $\left|\Pi_{2}(v) \cap \Pi_{1}(u)\right|=n / 2-1$; hence $c_{1}=1, b_{1}=n / 2-1$, and $a_{1}=n / 2-b_{1}-c_{1}=0$. Finally, if $u$ in $V(\Pi)$ and $\partial(u, v)=2$, then two vertices $u, v$ are not adjacent in $\Pi$, so $\left|\Pi_{1}(v) \cap \Pi_{1}(u)\right|=n / 2$; hence $c_{2}=n / 2$ and $a_{2}=n / 2-c_{2}=0$. So the intersection array of $\Pi$ is $\{n / 2, n / 2-1 ; 1, n / 2\}$. Therefore, by Theorem 4 , the tridiagonal (3) $\times(3)$ matrix

$$
\left[\begin{array}{ccc}
a_{0} & b_{0} & 0 \\
c_{1} & a_{1} & b_{1} \\
0 & c_{2} & a_{2}
\end{array}\right]=\left[\begin{array}{ccc}
0 & \frac{n}{2} & 0 \\
1 & 0 & \frac{n}{2}-1 \\
0 & \frac{n}{2} & 0
\end{array}\right]
$$

determines all the eigenvalues of $\Pi$. It is clear that all the eigenvalues of $\Pi$ are $n / 2,-n / 2,0$, and their multiplicities are $1,1, n-2$, respectively. So $\Pi$ is an integral graph.

Conclusion 10. Let $\Pi=\operatorname{Cay}\left(\mathbb{Z}_{n}, \Omega\right)$ be the Cayley graph on the cyclic group $\mathbb{Z}_{n}$ as before with the adjacency matrix $M=$ $\left[\begin{array}{cc}A & A \\ A & A\end{array}\right]$, and characteristic polynomial $\Phi_{\Pi}(x)$ then is

$$
\begin{aligned}
\Phi_{2 A}(x) & =\operatorname{det}\left(x I_{n / 2}-2 A\right) \\
& =\left(x-\frac{n}{2}\right)\left(x+\frac{n}{2}\right) x^{(n-4) / 2} .
\end{aligned}
$$

Proof. It is easy to show that the adjacency matrix $M=\left[\begin{array}{ll}A & A \\ A & A\end{array}\right]$, where $A$ is $n / 2 \times n / 2$ matrix; hence, by Proposition 9 and Theorem 5 ,

$$
\begin{aligned}
\Phi_{\Pi}(x) & =\operatorname{det}\left(x I_{n}-M\right)=\operatorname{det}\left[\begin{array}{cc}
x I_{n / 2}-A & -A \\
-A & x I_{n / 2}-A
\end{array}\right] \\
& =\operatorname{det}\left[\left(x I_{n / 2}-A\right)^{2}-A^{2}\right] \\
& =\operatorname{det}\left[\left(x I_{n / 2}-A\right)-A\right] \operatorname{det}\left[\left(x I_{n / 2}-A\right)+A\right] \\
& =\operatorname{det}\left(x I_{n / 2}-2 A\right) \operatorname{det}\left(x I_{n / 2}\right)=\Phi_{2 A}(x) x^{n / 2} \\
& =\left(x-\frac{n}{2}\right)\left(x+\frac{n}{2}\right) x^{(n-4) / 2} x^{n / 2} .
\end{aligned}
$$

Proposition 11. Let $\Lambda=\operatorname{Cay}\left(\mathbb{Z}_{n}, \Psi_{m}\right)$ be the Cayley graph on the cyclic group $\mathbb{Z}_{n}$ as before with the adjacency matrix $\widehat{M}$ and characteristic polynomial $\Phi_{\Lambda}(x)$. If $n \geq 8$ and $n / 2$ is an even integer, then

$$
\begin{aligned}
\Phi_{\Lambda}(x)= & \left(x-\left(\frac{n}{2}+1\right)\right)\left(x-\left(1-\frac{n}{2}\right)\right) \\
& \cdot(x-1)^{(n-4) / 2}(x+1)^{n / 2} .
\end{aligned}
$$

Proof. It is easy to show that the adjacency matrix $\widehat{M}=$ $\left[\begin{array}{cc}A & A+I \\ A+I & A\end{array}\right]$, where $A$ is $n / 2 \times n / 2$ matrix; hence, by Conclusion 10 and Theorem 5 ,

$$
\begin{aligned}
\Phi_{\Lambda} & (x)=\operatorname{det}\left(x I_{n}-\widehat{M}\right) \\
= & \operatorname{det}\left[\begin{array}{cc}
x I_{n / 2}-A & -(A+I) \\
-(A+I) & x I_{n / 2}-A
\end{array}\right]=\operatorname{det}\left[\left(x I_{n / 2}-A\right)^{2}\right. \\
& \left.-(A+I)^{2}\right]=\operatorname{det}\left[\left(x I_{n / 2}-A\right)\right. \\
& -(A+I)] \operatorname{det}\left[\left(x I_{n / 2}-A\right)+(A+I)\right] \\
& =\operatorname{det}\left[(x-1) I_{n / 2}-2 A\right] \operatorname{det}\left[(x+1) I_{n / 2}\right] \\
& =\Phi_{2 A}(x-1)(x+1)^{n / 2}=\left(x-\left(\frac{n}{2}+1\right)\right)(x \\
& \left.-\left(1-\frac{n}{2}\right)\right)(x-1)^{(n-4) / 2}(x+1)^{n / 2} .
\end{aligned}
$$

Conclusion 12. Let $\Lambda=\operatorname{Cay}\left(\mathbb{Z}_{n}, \Psi_{m}\right)$ be the Cayley graph on the cyclic group $\mathbb{Z}_{n}$, where $n$ is an even integer, $m=n / 2+1$, and $\Psi_{m}=\Omega \cup\{n / 2\}$ is the inverse-closed subset of $\mathbb{Z}_{n}-\{0\}$. If $n \geq 8$ and $n / 2$ is an even integer, then the adjacency matrix spectrum of $\Lambda$ is $(n / 2+1)^{1},(1-n / 2)^{1},(1)^{(n-4) / 2},(-1)^{n / 2}$.

\section{Competing Interests}

The authors declare that there is no conflict of interests regarding the publication of this paper. 


\section{References}

[1] C. Godsil and G. Royle, Algebraic Graph Theory, vol. 207 of Graduate Texts in Mathematics, Springer, New York, NY, USA, 2001.

[2] J. D. Dixon and B. Mortimer, "Permutation groups," Mathematical Proceedings of the Cambridge Philosophical Society, 1996.

[3] N. Biggs, Algebraic graph theory, Cambridge Mathematical Library, Cambridge University Press, Cambridge, Second edition, 1993.

[4] F. Harary and A. Schwenk, "Which graphs have integral spectra?" in Graphs and Combinatorics, vol. 406 of Lecture Notes in Mathematics, pp. 45-51, Springer, 1974.

[5] K. Balinska, D. Cvetković, Z. Rodosavljević, S. Simić, and D. Stevanović, "A survey on integral graphs," Univ. Beograd, Publ. Elektrotehn. Fak. Ser. Mat, vol. 13, pp. 42-65, 2002.

[6] O. Ahmadi, N. Alon, I. F. Blake, and I. E. Shparlinski, "Graphs with integral spectrum," Linear Algebra and its Applications, vol. 430, no. 1, pp. 547-552, 2009.

[7] A. Abdollahi and E. Vatandoost, "Which Cayley graphs are integral?” Electronic Journal of Combinatorics, vol. 16, no. 1, article R122, pp. 1-17, 2009.

[8] A. E. Brouwer, A. M. Cohen, and A. Neumaier, Distance-Regular Graphs, vol. 18, Springer, Berlin, Germany, 1989.

[9] J. R. Silvester, "Determinants of block matrices," The Mathematical Gazette, vol. 84, pp. 460-467, 2000.

[10] L. W. Beineke and R. J. Wilson, Eds., Topics in Algebraic Graph Theory, vol. 102 of Encyclopedia of Mathematics and its Applications, Cambridge University Press, Cambridge, UK, 2004. 


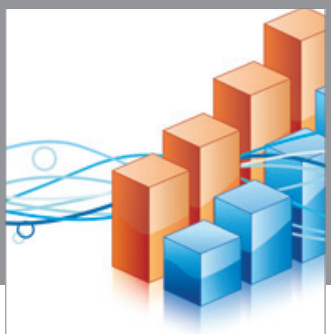

Advances in

Operations Research

vatem alat4

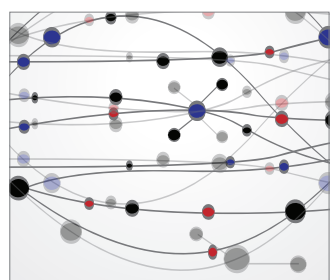

\section{The Scientific} World Journal
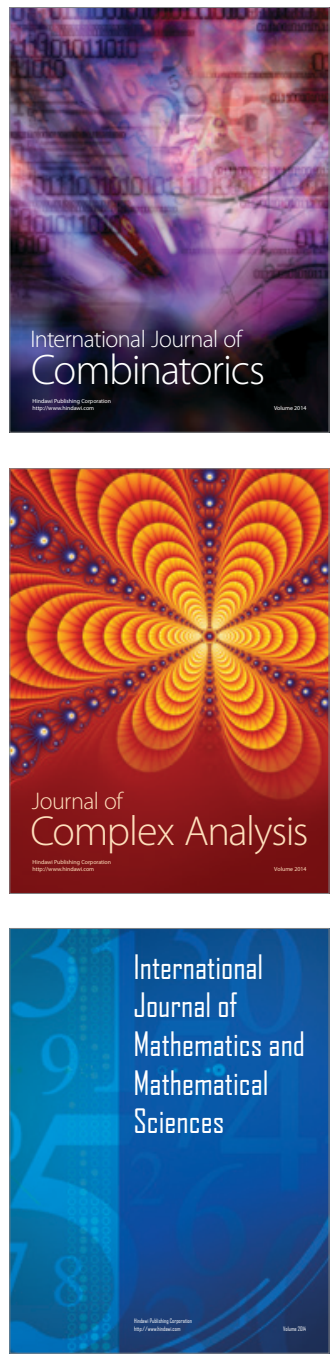
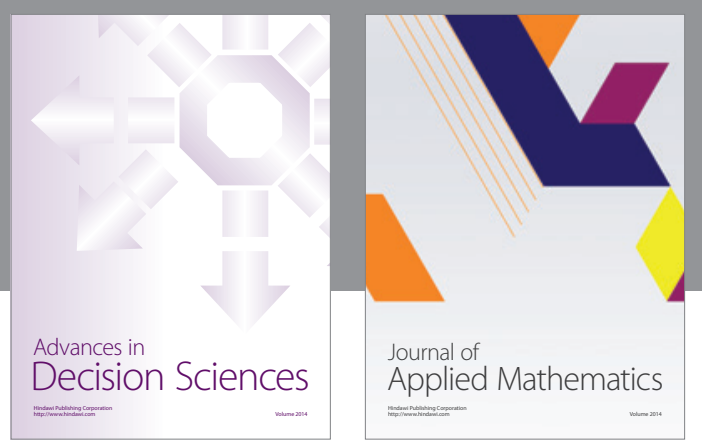

Algebra

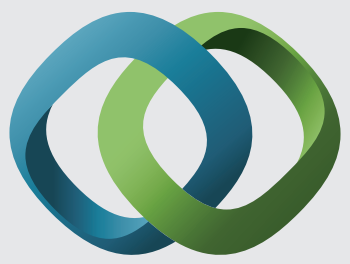

\section{Hindawi}

Submit your manuscripts at

https://www.hindawi.com
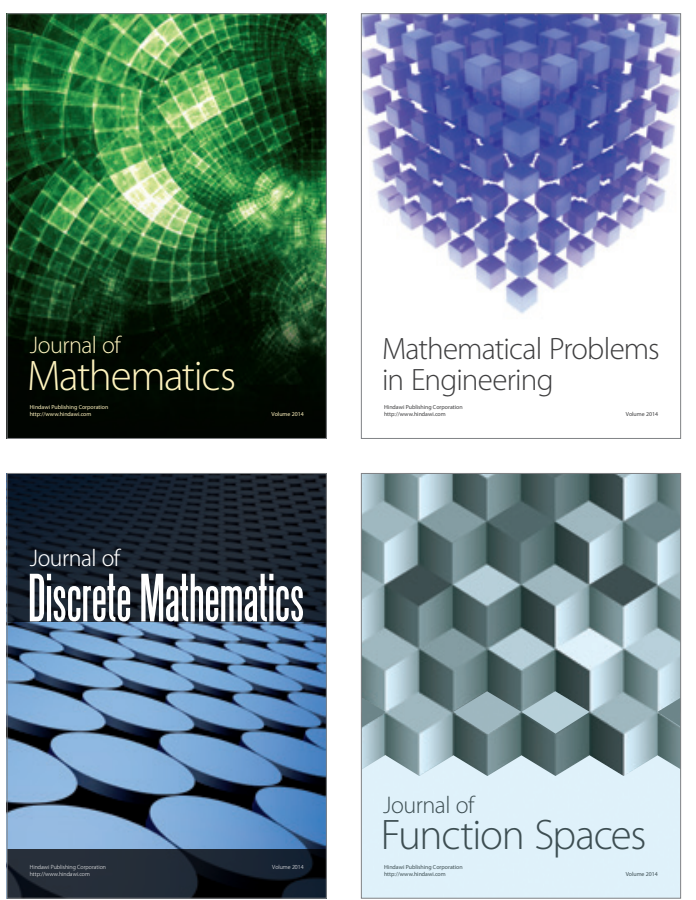

Mathematical Problems in Engineering
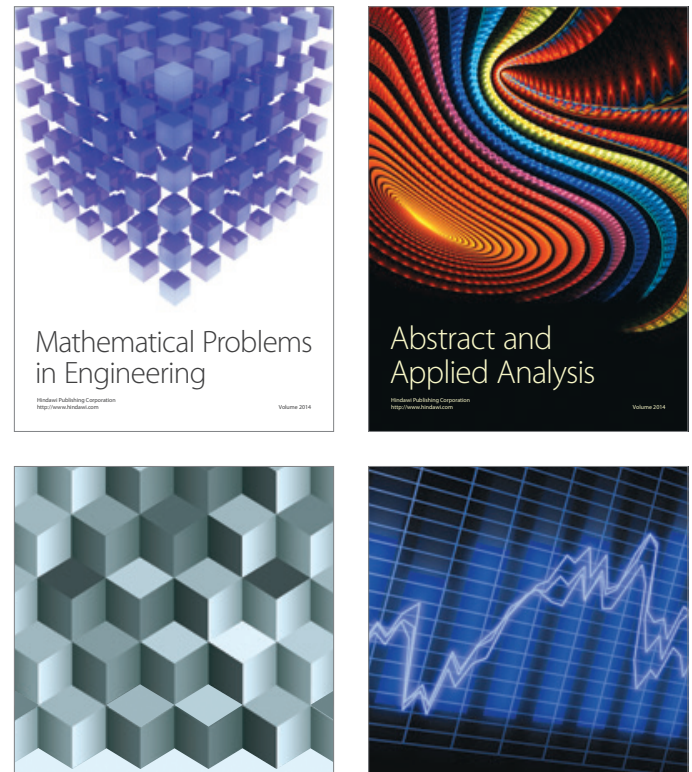

Journal of

Function Spaces

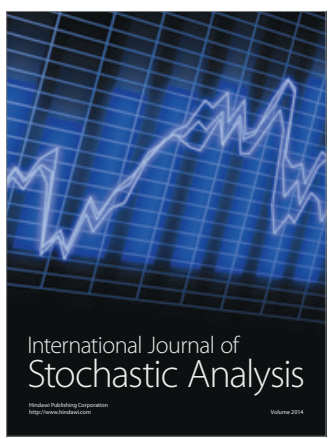

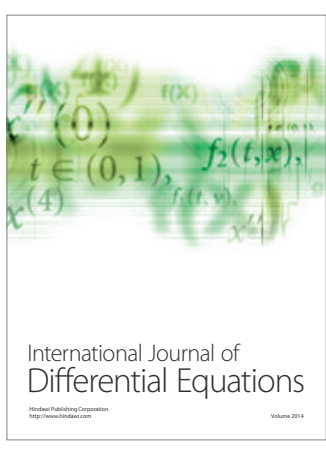
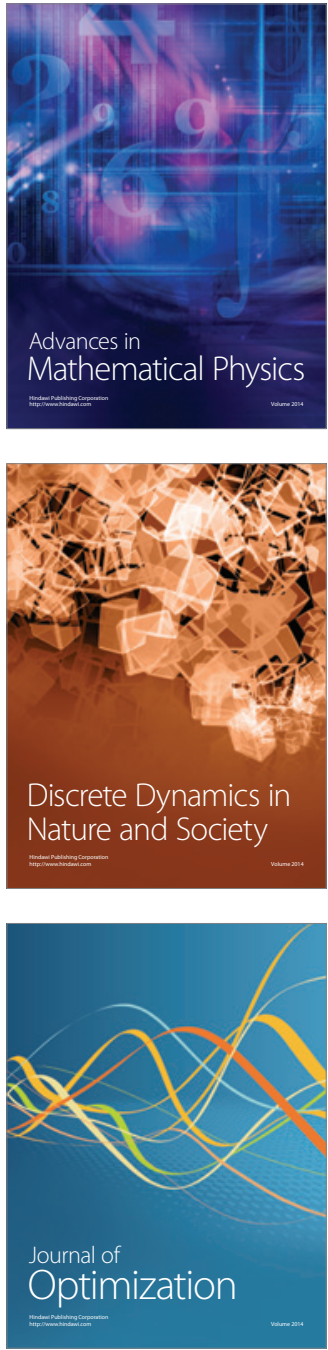